\title{
ОЦЕНКА ВЕРОЯТНОСТИ РОСТА ВРП В ЗАВИСИМОСТИ ОТ СТРУКТУРЫ ИНВЕСТИЦИЙ В ДАЛЬНЕВОСТОЧНОМ ФЕДЕРАЛЬНОМ ОКРУГЕ *
}

\author{
(c) 2021 Олейник Елена Борисовна \\ доктор экономических наук, доцент, профессор кафедры \\ Дальневосточный федеральный университет, Россия, Владивосток \\ e-mail: oleynik.eb@dvfu.ru
}

Современные условия развития экономики потребовали качественной оценки структуры инвестиций с целью ускорения роста объема ВРП. На основе логистической регрессии предлагается вероятностный подход и бинарный классификатор для оценки роста ВРП в зависимости от распределения инвестиционных вложений и пропорциональности структуры экономики. Полученные результаты могут быть использованы как вспомогательный инструмент инвестиционной политики Дальневосточного региона при принятии решений о направлении инвестиционных потоков.

Ключевые слова: распределение инвестиции, структурный сдвиг, бинарный классификатор, предельный эффект.

Социально-экономическое развитие Дальневосточного федерального округа (ДВФО) статистически характеризуется стабильной динамикой ключевых макропоказателей с признаками неустойчивости в отдельных сферах деятельности [4]. ВРП макрорегиона демонстрирует устойчивый рост, а приток инвестиций достаточно стабилен (рис. 1).

Однако субъекты Дальневосточного федерального округа значительно отличаются друг от друга по уровню инвестиций в основной капитал в расчете на душу населения. Территории с наиболее высокой долей добывающей промышленности в структуре ВРП и невысокой плотностью населения, такие как Сахалинская и Магаданская области, Республика Саха (Якутия), Чукотский автономный округ, привлекают больший объем частных инвестиций на душу населения. В регионах без значимого промышленного сектора бюджетные инвестиции зачастую формируют существенную часть общего объема инвестиций. К таким регионам относятся, например, Республика Бурятия, Камчатский край. Средний уровень бюджетных инвестиций (из бюджетов всех уровней, включая федеральный) в ДВФО соответствует среднероссийскому показателю. Однако в связи с неравномерным распределением бюджетных инвестиций, более

* Работа подготовлена в рамках гранта РФФИ № 19-010-00085 «Комплексная оценка структурной динамики экономической системы Дальневосточного региона на основе моделирования результатов стратегических инвестиционных проектов»

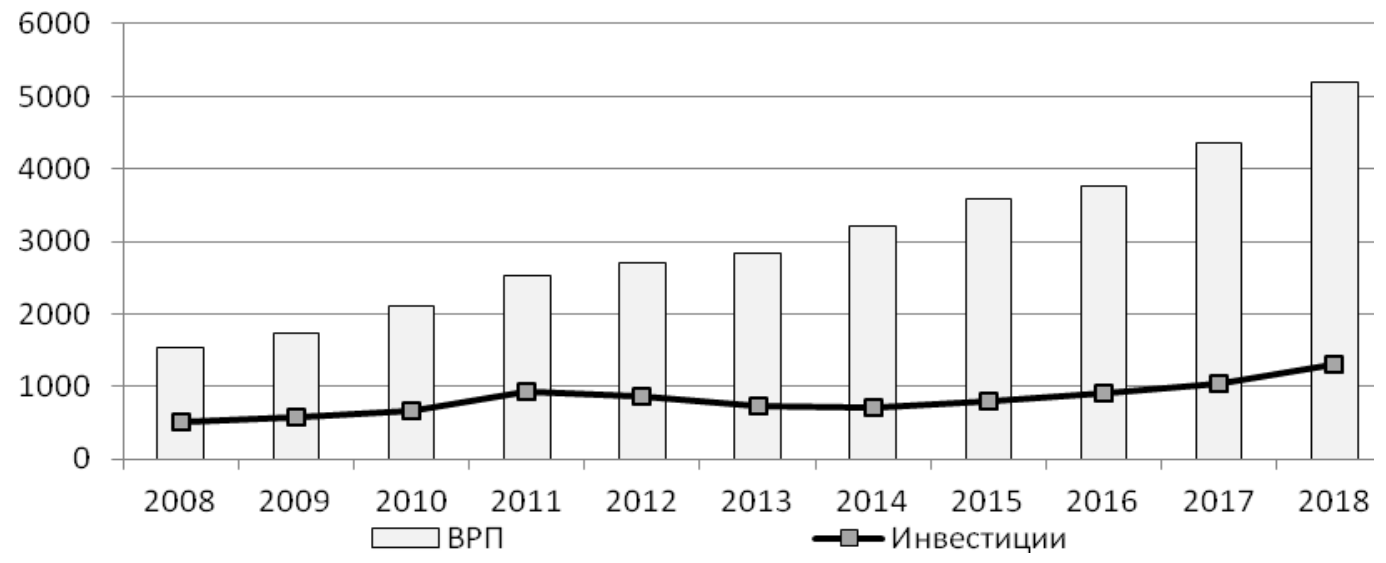

Puc. 1. ВРП и инвестиции в основной капитал ДВФО, млрд. руб. Источник: https://rosstat.gov.ru/folder/512 
высокой стоимостью жизни, а также ограниченной транспортной доступностью территорий макрорегиону требуется значительно больший объем инвестиций [5]. Современные условия развития экономики потребовали качественной оценки структуры инвестиций с целью ускорения роста объема ВРП. Такую оценку можно получить с помощью эконометрического моделирования зависимости роста объемов ВРП от структуры инвестиций и сдвигов в структуре экономики.

Сформулируем гипотезу исследования: пропорциональность структуры экономики и преимущественное перераспределение инвестиций в обрабатывающую промышленность и социальную сферу и инфраструктуру увеличивают вероятность роста ВРП в Дальневосточном регионе.

Методологический подход к исследованию. В работах $[2,10]$ и других описаны логистические регрессии, которые применяются для построения моделей бинарного выбора. Подобную логистическую панельную регрессию мы и будем использовать для оценки вероятности роста доли субъекта в ВРП и построим уравнение (1).

$$
\frac{P(Y i=1)}{P(Y i=0)}=\exp \left(\alpha_{0}+\alpha_{1} * X_{i_{1}}+\alpha_{2} * x_{i 2}+\cdots+\alpha_{n} * x_{i n}\right)
$$

где $Y$ - эндогенная дамми-переменная, которая принимает значение 1 , если наблюдался положительный прирост доли субъекта в ВРП региона и 0 - иначе; $X=\left(x_{1}, \ldots, x_{n}\right)-$ матрица значений экзогенных экономических переменных в каждом субъекте региона; $\alpha=\left(\alpha_{1}, \ldots, \alpha_{n}\right)$ - вектор неизвестных параметров, оцениваемых с помощью метода максимального правдоподобия.

С помощью уравнения (1) мы сможем определить вероятность, с которой зависимая переменная $Y$ примет значение равное 1 при некоторых заданных факторах $X$. Величина $P\left(Y_{i}=1\right) / P\left(Y_{i}=0\right)$ является шансами события $\left\{Y_{i}=1\right\}$, что соответствует росту ВРП.

При каком значении вероятности будем полагать, что получен положительный прирост ВРП? Для этого нам нужна бинарная классификация. Проведение ROC-анализа для бинарной классификации, на основе логистических регрессий представлено в работах [6, 9, 11]. Необходимо рассчитать порог отсечения - критерий классификации: если полученная вероятность выше порога отсечения, будем считать, что произошло событие $\left\{Y_{i}=1\right\}$, то есть имеем рост ВРП. В противном случае будем считать, что произошло событие $\left\{Y_{i}=0\right\}$, то есть рост ВРП не наблюдается. Чтобы классифицировать вероятностный результат, полученный с помощью модели логистической регрессии, будем использовать анализ receiver operating characteristic (ROC-анализ).

Основой ROC-анализа является построение ROC-кривой на основании результатов логит-регрессии. ROC-кривая характеризует зависимость количества (доли) верно классифицированных положительных результатов (эту величину называют чувствительностью, Se) от количества (доли) ложноположительных результатов, что часто записывается, как 1-Sp, где $\mathrm{Sp}$-специфичность, то есть доля верно классифицированных отрицательных случаев. Количественную интерпретацию ROC даёт показатель AUC (areaunder ROC-curve). Чем выше показатель AUC, тем качественнее классификатор. В соответствии со шкалой экспертных оценок [1] значение 0,5 демонстрирует непригодность выбранного метода классификации.

Определим оптимальный порог отсечения с помощью индекса Йодена (формула 2), как максимальное отличие ординат ROC-кривой и диагонали $\mathrm{Se}=1-\mathrm{Sp}[12]$ :

$$
\mathrm{J}=\max (\mathrm{Se}+\mathrm{Sp}-1)
$$

Если полученная вероятность превышает порог отсечения, то мы полагаем $\left\{Y_{i}=1\right\}$, в противном случае $\left\{Y_{i}=0\right\}$.

Описание переменных логистической регрессии. В результате исключения незначимых факторов и факторов, имеющих неинтерпретируемые знаки, в модель логистической панельной регрессии были включены следующие переменные: year - год наблюдения; ID - индексная переменная, которая используется для организации панели и соответствует следующим субъектам ДВФО: 1 - Якутия; 2 - Камчатская область; 3 - Приморский край; 4 - Хабаровский край; 5 - Амурская область; 6 - Магаданская область; 7 - Сахалинская область; 8 - Еврейская АО; 9 - Чукотский АО. Все остальные переменные представлены для каждого из 9 субъектов ДВФО:

D_GRP - дамми-переменная, принимающая значение 1 , что соответствует приросту доли субъекта в ВРП региона и 0 - иначе; Sdv kv_GRP - квадратичные сдвиги в структуре ВРП 
каждого субъекта региона (формула 3), измеряется в долях.

$$
S_{\text {относ }}=\sqrt{\sum_{j=1}^{n} \frac{\left(f_{j}^{l}-f_{j}^{l-1}\right)^{2}}{f_{j}^{l-1}}},
$$

где $f_{j}^{l}$ - доля $j$-го элемента структуры в периоде $l ; f_{j}^{l-1}$ - доля $j$-го элемента структуры в периоде $l-1 ; n-$ число элементов структуры. В научной литературе [3,7,8 и других] описаны различные показатели структурных изменений в экономике. В данной статье рассмотрим только квадратичный коэффициент относительных структурных сдвигов, который характеризует равномерность изменения удельных весов в динамике и поэтому может быть использован для отражения во времени пропорциональности развития структуры. Этот коэффициент показывает, на сколько, в среднем отклоняются коэффициенты (или темпы) роста отдельных частей совокупности от их среднего значения, или иначе: какова средняя квадратичная величина относительного отклонения удельных весов;

IND_quality - индекс качества структуры инвестиций (формула 4), измеряется в долях. Структуру привлеченных инвестиций будем рассматривать в разрезе отраслей экономики, и через направление инвестиционных вложений определим качество структуры инвестиций.

$$
I N D_{\text {quality }}=\frac{I n v_{O b r}+I n v_{Z d r}+I n v_{O b r a Z}+I n v_{I n f r}}{\operatorname{In} v_{D o b}}
$$

где Inv $v_{\mathrm{Obr}}$ - инвестиции в обрабатывающую промышленность, Inv $v_{Z d r}-$ инвестиции в здравоохранение, Inv $v_{\text {ObraZ }}$ - инвестиции в образование, Inv ${ }_{\text {Infr }}$ - инвестиции в развитие инфраструктуры; Inv $v_{D o b}$ инвестиции в добывающую промышленность.

Чем больше единицы этот индекс, тем более качественной будет структура инвестиций. Так как экономика ДВФО имеет ресурсную направленность, то показателем качества структуры инвестиций является ее сбалансированность: превышение доли инвестиций в социальную сферу, инфраструктуру и обрабатывающие отрасли промышленности над добывающими отраслями. Соотношение инвестиций, представленное в формуле (4) в перспективе должно снизить миграцию населения в центр из регионов.

С помощью такого набора переменных мы учитываем динамику и пропорциональность развития структуры экономики.

Описание исходных данных и результатов. Исходными данными для моделирования послужили статистические выборки за период с 2006 по 2019 год из официальных источников*. Для оценки влияния квадратичных сдвигов предварительно были рассчитаны доли отраслей в структуре ВРП, а также индекс качества инвестиций для каждого из девяти субъектов Дальневосточного региона.

Расчет параметров модели произведен с помощью эконометрического пакета Gretl. Peзультаты представлены в таблице 1. Зависимой в этом уравнении является дамми-переменная D_GRP.

Уравнение логистической регрессии выглядит так:

$$
\begin{aligned}
\frac{P\left(\mathbf{D}_{\mathrm{GRP} i}=\mathbf{1}\right)}{P\left(\mathrm{D}_{\mathrm{GRP} i}=\mathbf{0}\right)}==\exp ( & -\mathbf{0 . 6 8 7 9 2 7}+\mathbf{0 . 3 8 3 2 2 9} * \mathbf{S d v}_{\mathrm{kv}_{\mathrm{GRP}_{i}}}+ \\
& \left.+\mathbf{0 . 0 0 0 9 9 1 2 7 1} * \mathrm{IND}_{\text {quality }_{i}}\right)
\end{aligned}
$$

Все коэффициенты регрессии являются значимыми. Константа является значимой и отрицательной, следовательно, существуют и другие, достаточно влиятельные факторы, которые снижают вероятность роста ВРП. Но эти факторы остаются за рамками нашего исследования. Коэффициент при переменной Sdv_kv_GRP значим на уровне 5\%, а при переменной IND_quality_1 на уровне $10 \%$. Знаки коэффициентов положительные, следовательно, увеличение значений факторных переменных увеличивает в целом вероятность роста ВРП, так как сами переменные не могут быть отрицательными, что подтверждает нашу гипотезу. Рассчитанное значение параме-

\footnotetext{
* Данные Федеральной службы государственной статистики https://rosstat.gov.ru/regional_statistics; Министерства Российской Федерации по развитию Дальнего Востока и Арктики https://minvr.gov.ru/opendata; Управления федеральной службы государственной статистики по Приморскому краю https:/primstat.gks.ru/ statistic; Управления федеральной службы государственной статистики по Хабаровскому краю, Магаданской области, Еврейской автономной области и Чукотскому автономному округу https://habstat.gks.ru/; данные о TOP https://rosinfostat.ru/territorii-operezhayushhego-razvitiya.
} 
Таблица 1. Логистическая регрессия

Model 3: Logit, using 126 observations

Dependent variable: D_GRP

QML standard errors

\begin{tabular}{|l|c|c|c|c|}
\hline & Coefficient & Std. Error & $z$ & $p$-value \\
\hline Const & -0.687927 & 0.329331 & -2.0889 & 0.0367 \\
\hline Sdv_kv_GRP & 0.383229 & 0.195392 & 1.9613 & 0.0498 \\
\hline IND_quality & 0.000991271 & 0.00052341 & 1.8939 & 0.0582 \\
$*$
\end{tabular}

\begin{tabular}{|l|r|}
\hline Meandependentvar & 0.468254 \\
\hline McFadden R-squared & 0.030877 \\
\hline Log-likelihood & -84.39359 \\
\hline Schwarzcriterion & 183.2960 \\
\hline
\end{tabular}

\begin{tabular}{|l|r|}
\hline S.D. dependentvar & 0.500983 \\
\hline Adjusted R-squared & -0.003573 \\
\hline Akaikecriterion & 174.7872 \\
\hline Hannan-Quinn & 178.2441 \\
\hline
\end{tabular}

Number of cases 'correctly predicted' $=72(57.1 \%)$

$\mathrm{f}($ beta' $\mathrm{x})$ at mean of independent vars $=0.501$

Likelihood ratio test: Chi-square $(2)=5.37763$ [0.0680]

тра AUC=0.61, следовательно, в соответствии со шкалой экспертных оценок [1] качество модели удовлетворительное.

В этой модели в правую часть включена дамми-переменная D_GRP, которая принимает значение 1, если в $i$-том году был получен положительный прирост ВРП и значение 0 в противном случае. Коэффициенты модели логистической регрессии интерпретируются как отношение шансов. Уравнение (5) может быть использовано для оценки шансов наступления события D_GRP=1. Зависимая переменная D_GRP показывает вероятность того, что прирост ВРП будет положительным. С увеличением квадратичного структурного сдвига на $1 \%$, шансы роста ВРП возрастут в $\exp (0.383229) \approx 1.5$ раза. Так как коэффициент при переменной IND_quality_1 достаточно мал, мы можем интерпретировать рост ВРП в процентах, то есть с увеличением индекса качества структуры в предыдущем году на $1 \%$, шансы роста ВРП возрастут на $0,1 \%$, то есть не уменьшаются и будут достаточно стабильны. Легче интерпретировать полученные значения в терминах предельных эффектов. Оценим вероятность роста ВРП с помощью предельных эффектов - частных производных в средних значениях факторов. Среднее значение квадратичного сдвига равно 1.4158, предельный эффект составил $178 \%$; в среднем вероятность роста ВРП возрастет в 1.78 раза с увеличением квадратичного сдвига на $1 \%$. Среднее значение индекса качества структуры инвестиций равно
3.264, предельный эффект составил 0,4\%; в среднем вероятность роста ВРП возрастет на $0,4 \%$ с увеличением индекса качества на $1 \%$.

С помощью модели можно прогнозировать вероятность того, что будет получен рост ВРП, что не очень удобно при принятии решений. Возникает вопрос: при каком значении величины отношения шансов $P\left(Y_{i}=1\right) / P\left(Y_{i}=0\right)$ можно считать, что мы получим положительный прирост ВРП? Нам нужна бинарная классификация: есть или нет прирост ВРП при распределении инвестиций, которое задается с помощью индекса качества.

Определим критерий классификации, то есть рассчитаем порог отсечения. Это максимальное отличие ординат ROC-кривой и диагонали. Соответствующее ему расчетное значение на оси абсцисс равно 0.64. Также в R Studio c использованием пакета $\mathrm{pROC}$ рассчитаем порог отсечения как абсциссу точки пересечения кривых чувствительности и специфичности. Соответствующее ему расчетное значение на оси абсцисс равно 0.47. Проинтерпретируем полученные результаты (рис. 2).

При вероятности D_GRP $<0.47$, считаем, что D_GRP $=0$. В этом случае имеем максимальный риск того, что реализуемые инвестиционные проекты не дадут положительного прироста ВРП и надо перераспределить инвестиции между инвестиционными проектами так, чтобы индекс качества структуры был больше 1 . 


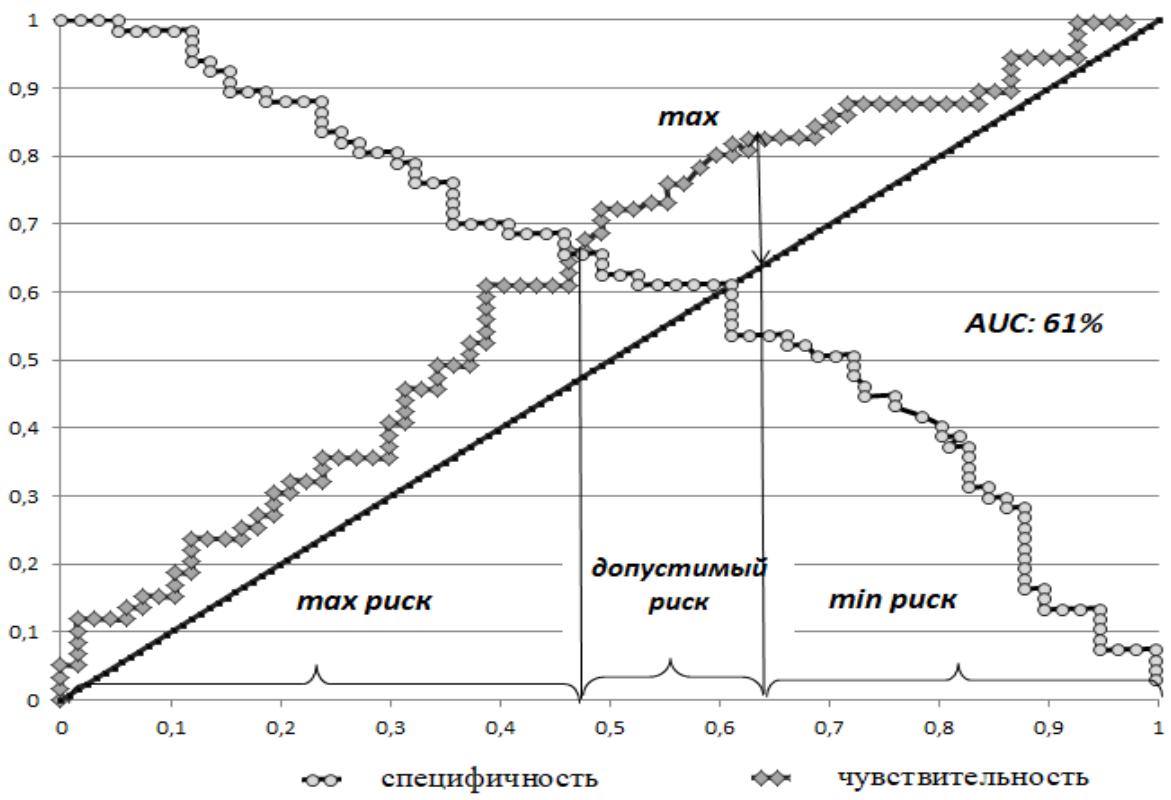

Рис.2 ROC-анализ Logit-модели

На интервале $0.47 \leqslant D_{-} \mathrm{GRP} \leqslant 0.64$ имеем допустимый риск того, что распределение инвестиций, заданное индексом качества структуры, не приведет к росту GRP. В этом случае нужно провести дополнительный анализ инвестиционных проектов, которые предполагается реализовать на территории ДВФО.

При вероятности D_GRP $>0.64$ считаем, что D_GRP=1. В этом случае предполагаем, что получим положительный прирост ВРП, и с минимальным риском ошибки можно предполагать, что реализуемые инвестиционные проекты будут удачно завершены и будет наблюдаться рост ВРП в макрорегионе.

Выводы и заключение. В своем исследовании мы оценили вероятность роста ВРП при заданной структуре инвестиций, выдвинули и подтвердили с помощью логистической регрессии гипотезу о том, что на рост ВРП в Дальневосточном регионе положительное влияние оказывает пропорциональность структуры экономики, вызванная преимущественным распределением инвестиций в обрабатывающие отрасли промышленности, инфраструктуру и социальную сферу. Предложенный подход оценки связи роста ВРП и распределения инвестиций между отраслями экономики, а также разработанный критерий бинарной классификации может являться вспомогательным инструментом инвестиционной политики Дальневосточного региона при принятии решений о направлении инвестиционных потоков.

\section{Библиографический список}

1. Ковалев А. А., Кузнецов Б. К., Ядченко А. А., Игнатенко В. А. Оценка качества бинарного классификатора в научных исследованиях //Проблемы здоровья и экологии, 2020. -№ 4 (66). - С. 105-113

2. Матраева Л.В.Использование логистической регрессии при выявлении приоритетов региональной инвестиционной политики в отношении иностранных инвесторов в регионы РФ //Экономика, статистика и информатика. Вестник УМО. 2013. № 6. С.170-174

3. Олейник Е.Б., Захарова А.П., Юрченко Е.Г. Комплексный анализ сдвигов в структуре инвестиций Дальневосточного региона// Экономические науки, 2020. -№ 190. - С. 119-124

4. Першин М., Анисимова Е. ДФО - долгосрочно финансировать облигациями: источники бюджетных инвестиций для регионов Дальневосточного федерального округа // Исследование Аналитического кредитного рейтингового агентства (АКРА) [Электронный ресурс] https://roscongress.org/upload/medialibrary/345/fareast-federal-district-_-long_term-debt-financing-needed.pdf (дата обращения: 11.11.2021) 
5. Прокапало О.М., Бардаль А. Б., Исаев А. Г., Мазитова М. Г., Суслов Д. В.. Экономическая конъюнктура в Дальневосточном федеральном округе в 2019 г.// Пространственная экономика, 2020. - т. 16.- № . 2. - С. $142-184$

6. Старовойтов В. В., Голуб Ю. И. Сравнительный анализ оценок качества бинарной классификации //Информатика, 2020. - Т.17. - № 1. С. 87-101.

7. Сухарев О.С. Экономический рост и технологическое обновление: структурная динамика // Journal of New Economy, 2019. -T. 20. -№ 2. -С. 30-54.

8. Узяков Р. М. Метрики структурных сдвигов и необходимость учета межотраслевых связей// Проблемы прогнозирования, 2020.- № 2. -С. 25-35.

9. Fawcett T. An Introduction to ROC analysis // Pattern Recognition Letters. 2006. No 27 P. 861-874.

10. Hosmer D. W., Lemeshow S. Applied Logistic Regression. 2nd edn. Wiley, New York, 2000

11. Powers D. Evaluation: From Precision, Recall and F-Measure to ROC, Informedness, Markedness \& Correlation. Journal of Machine Learning Technologies, 2011, no. 2 (1), pp. 37-63.

12. Youden, W. J. Index for rating diagnostic tests // Cancer. 1950. Vol.3, Issue1. pp. 32-35. 